\title{
Agomelatin add-on bringt keine Vorteile
}

Fragestellung: Führt eine Kombination von Agomelatin zusätzlich zu einem bereits eindosierten Stimmungsstabilisierer (Lithium oder Valproat) zu einer Verbesserung der depressiven Symptome bei Bipolar-I-Patienten?

Hintergrund: Bipolare Depressionen sind meist schwer und langwierig. Nur wenige Medikamente sind zur Behandlung zugelassen. Agomelatin als Antidepressivum mit einem neuartigen pharmakologischen Profil (MT1- und MT2-Rezeptoragonist und $5-\mathrm{HT}_{2 \mathrm{c}}$-Rezeptorantagonist) hatte bei unipolarer Depression einen antidepressiven Effekt gezeigt, sodass nun seine Wirksamkeit gegenüber Placebo als Kombination mit einem Stimmungsstabilisierer geprüft werden sollte.

Patienten und Methodik: Die internationale, doppelblinde, randomisierte Multicenterstudie schloss insgesamt 344 Patienten ein, die bereits aufgrund einer depressiven Episode bei Bipolar-I-Erkrankung mit Lithium oder Valproat in therapeutisch wirksamer Dosierung seit mindestens sechs Wo-

Yatham LN, Vieta E, Goodwin GM et al; Agomelatine Study Group. Agomelatine or placebo as adjunctive therapy to a mood stabiliser in bipolar I depression: randomised double-blind placebo-controlled trial. Br J Psychiatry 2016; $208: 78-86$ chen behandelt worden waren. Nach der Stratifizierung für den Stimmungsstabilisierer erhielten die Patienten randomisiert verblindet 25 mg Agomelatin oder Placebo über acht Wochen. Bei fehlender Wirksamkeit konnte eine Erhöhung auf $50 \mathrm{mg}$ Agomelatin erfolgen, auch hierfür waren Behandler und Patienten verblindet. Anschließend erfolgte eine Weiterbeobachtung über einen Zeitpunkt von zehn Monaten. Primärer Endpunkt war eine Reduktion in der Montgomery-Åsberg Depression Rating Scale (MADRS). Sekundäre Endpunkte waren Response (Reduktion um $\geq 50 \%$ in der MADRS) sowie Änderungen in weiteren standardisierten Fragebögen.

Ergebnisse: 172 Patienten erhielten Agomelatin und 172 Placebo zusätzlich zum Stimmungsstabilisierer. Die Reduktion der depressiven Symptome im MADRS war nach acht Wochen in beiden Gruppen klinisch relevant, aber nicht signifikant unterschiedlich mit - 15,4 Punkten in der Verum- und - 15,2 Punkten in der Placebogruppe. Entsprechend kam es zu fast identischen Responseraten (61,9\% Verum und 60,8\% Placebo, p = $0,837)$. Die Remissionsrate ( $\leq 12$ im MADRS) in der Verumgruppe lag bei $53 \%$, in der Placebogruppe bei 53,2\%. 244 Patienten wurden über weitere zehn Monate beobachtet. Auch hier zeigten sich keine Unterschiede zwischen den beiden Gruppen. Auch bei allen weiteren sekundären Endpunkten zeigten sich keine signifikanten Gruppenunterschiede, ebenso bei den unerwünschten Wirkungen.

Schlussfolgerungen: Agomelatin war Placebo in der Akutbehandlung einer Bipolar-I-Depression nicht überlegen. Auch im Nachbeobachtungszeitraum von zehn Monaten zeigte sich kein Vorteil von Agomelatin bei der Erhaltungstherapie.

\section{- Kommentar von Alice Engel, Mainz}

\section{Auch bei bipolarer Depression nicht ausreichend antidepressiv wirksam}

Es handelt sich hierbei um die erste publizierte doppelblinde, randomisierte klinische Multicenterstudie zum Vergleich von Agomelatin und Placebo als Kombinationstherapie mit Lithium oder Valproat bei Bipolar-I-Depression. Sie erreicht eine hohe Qualität mit fünf von fünf Jadad-Punkten. Es zeigte sich jedoch keine Überlegenheit von Agomelatin gegenüber Placebo.

Auffällig waren die unerwartet hohen Responseraten in beiden Gruppen. Frühere Studien hatten Responseraten zwischen 27,3\% und $42 \%[2,3]$. Eine Metaanalyse von 2012 zeigte, dass Studien mit Responseraten über $40 \%$ bei Placebo wenig Aussagekraft über einen Wirksamkeitsunterschied hatten [1]. Die Autoren vermuteten zudem, dass die Punktezahl im MADRS mancher Patienten erhöht wurde, um ihnen die Teilnahme an der Studie zu ermöglichen, aber auch nach einer Post-hocAnalyse, die Zentren mit zu hoher Placeboresponse ausschloss, konnte kein signifikanter Vorteil von Agomelatin gefunden werden.

Die Studie unterstützt den klinischen Eindruck und auch die Metaanalysen bei unipolarer Depression, dass Agomelatin we- nig Effektivität bei der Behandlung von bipolarer Depressionen besitzt. Weiterhin gibt es somit wenige Therapieoptionen für diese schwerwiegende Erkrankung.
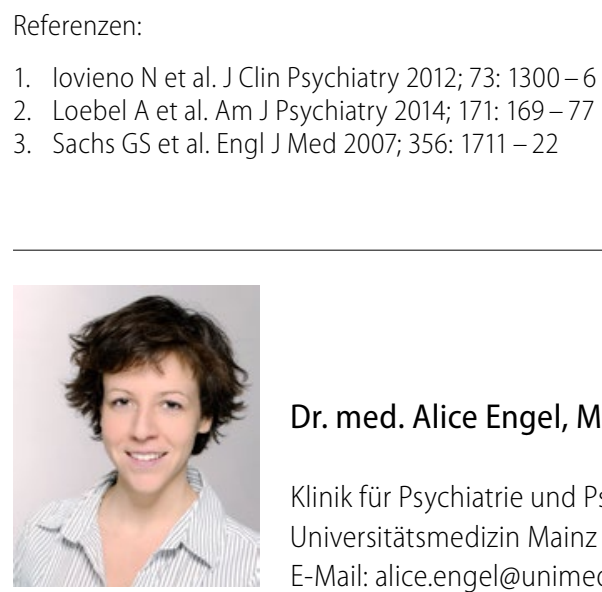

Dr. med. Alice Engel, Mainz

Klinik für Psychiatrie und Psychotherapie, Universitätsmedizin Mainz E-Mail: alice.engel@unimedizin-mainz.de 